\title{
Multi-band properties of three unidentified INTEGRAL sources
}

\section{R. Landi}

INAF/IASF Bologna, Via P. Gobetti 101, 40129 Bologna, Italy E-mail: Landiliasfbo.inaf.it

\section{J. B. Stephen}

INAF/IASF Bologna, Via P. Gobetti 101, 40129 Bologna, Italy

E-mail: stephen@iasfbo.inaf.it

\section{N. Masetti}

INAF/IASF Bologna, Via P. Gobetti 101, 40129 Bologna, Italy

E-mail: masettiliasfbo.inaf.it

\section{Sguera*}

INAF/IASF Bologna, Via P. Gobetti 101, 40129 Bologna, Italy

E-mail: sgueradiasfbo.inaf.it

\section{Grupe}

Department of Astronomy and Astrophysics, Pennsylvania State University, University Park, PA 16802

E-mail: grupedastro.psu.edu

\section{F. Capitanio}

INAF/IASF Roma, via Fosso del Cavaliere 100, 00133 Roma, Italy

E-mail: fiamma.capitaniodiasf-roma.inaf.it

\section{A. J. Bird}

School of Physics and Astronomy, University of Southampton, Highfield, SO17 1BJ, UK

E-mail: ajdephys.soton.ac.uk

Thanks to follow-up observations performed with the X-ray telescope (XRT) on board Swift several new unidentified hard X-ray sources revealed by INTEGRAL heve been localized with a positional accuracy of few arcseconds, thus allowing a more efficient and reliable search for optical, UV, infrared and radio counterparts. Here we provide spectral information over a broad range of frequencies for three still unclassified INTEGRAL objects: IGR J18249-3243, IGR J19443+2117 and IGR J22292+6647. Overall, their optical, UV, infrared and radio characteristics together with their X-/gamma-ray properties, point to an AGN classification for all three sources.

7th INTEGRAL Workshop

September 8-11 2008

Copenhagen, Denmark

\footnotetext{
* Speaker.
} 
Table 1: XRT positions of the three unidentified INTEGRAL sources.

\begin{tabular}{lccc}
\hline \hline Source & $\begin{array}{c}\text { R.A. } \\
(\mathrm{J} 2000)\end{array}$ & $\begin{array}{c}\text { Dec } \\
(\mathrm{J} 2000)\end{array}$ & $\begin{array}{c}\text { Error } \\
(\operatorname{arcsec})\end{array}$ \\
\hline \hline IGR J18249-3243 & 182456.11 & -324258.9 & 3.7 \\
\hline IGR J19443+2117 & 194356.20 & +211822.9 & 3.5 \\
\hline IGR J22292+6647 & 222913.50 & +664651.8 & 3.6 \\
\hline \hline
\end{tabular}

Table 2: XRT/IBIS broad-band best-fit parameters.

\begin{tabular}{lccccccc}
\hline \hline Source & $N_{\mathrm{H}(\mathrm{Gal})}^{a}$ & $N_{\mathrm{H}}^{a}$ & $\Gamma$ & $C_{\text {calib }}^{b}$ & $\chi^{2} / v$ & $F_{(2-10 \mathrm{keV})}^{c}$ & $F_{(20-100 \mathrm{keV})}^{c}$ \\
& & & & & & & \\
\hline \hline IGR J18249-3243 & 0.118 & - & $1.54 \pm 0.12$ & $0.52_{-0.21}^{+0.35}$ & $32.7 / 31$ & $0.52 \pm 0.03$ & $0.79 \pm 0.15$ \\
\hline IGR J19443+2117 & 0.836 & $0.54_{-0.13}^{+0.12}$ & $2.04 \pm 0.12$ & $0.60_{-0.28}^{+0.37}$ & $84.3 / 93$ & $1.83 \pm 0.04$ & $1.12 \pm 0.22$ \\
\hline IGR J22292+6647 (low) & 0.481 & - & $1.60 \pm 0.10$ & $0.81_{-0.34}^{+0.45}$ & $63.3 / 68$ & $0.53 \pm 0.02$ & $1.10 \pm 0.02$ \\
IGR J22292+6647 (high) & 0.481 & - & $1.66 \pm 0.14$ & $0.60_{-0.26}^{+0.42}$ & $52.7 / 59$ & $0.85 \pm 0.03$ & $1.10 \pm 0.02$ \\
\hline \hline
\end{tabular}

${ }^{a}$ In units of $10^{22} \mathrm{~cm}^{-2}$ (from Kalberla et al. (2005));

${ }^{b}$ IBIS/XRT cross-calibration constant;

${ }^{c}$ In units of $10^{-11} \mathrm{erg} \mathrm{cm}^{-2}$.

\section{IGR J18249-3243}

Within the XRT positional uncertainty (see Table 1) there is a counterpart in the USNO-B1.0 catalogue (Monet et al. 2003), with magnitude $R$ in the range 14.6-14.8, which is also listed in the 2MASS survey (Skrutskie et al. 2006) with magnitudes $J=13.456 \pm 0.026, H=13.007 \pm$ 0.029 and $K=12.675 \pm 0.022$, which is also detected with Swift/UVOT in $U V W 2=17.53 \pm$ 0.02 . The Galactic colour excess along the line of sight, $E(B-V)=0.23$, estimated here and afterwards following Schlegel et al. (1998), indicates a Galactic column density of $0.13 \times 10^{22}$ $\mathrm{cm}^{-2}$ (Predehl \& Schmitt 1995), which is compatible with the value reported in Table 2 (estimated following Kalberla et al. 2005). Recently, optical follow-up observations of IGR J18249-3243 have confirmed the AGN nature of this source despite difficulties in separating the active galaxy at $z=0.355$ from a foreground star (Masetti et al. in prep.), which likely contaminates the USNOB1.0, 2MASS and UV magnitudes. However, we are able to provide an upper limit to the R flux of $7.6 \times 10^{-12} \mathrm{erg} \mathrm{cm}^{-2} \mathrm{~s}^{-1}$, adopting the conversion factor of Fukugita et al. (1995).

The Swift/XRT and INTEGRAL/IBIS broad-band spectrum (shown in Figure 1) is well described by a simple power law absorbed by Galactic absorption (see Table 2 ) having a flat $(\Gamma \sim 1.5$ ), but still within the range observed in AGNs.

Figure 2 (left) shows the radio image taken from the NVSS (NRAO VLA Sky Survey, Condon et al. 1998) at $1.4 \mathrm{GHz}$ with superimposed the IBIS uncertainty circle and the XRT position. This radio counterpart, which is coincident within uncertainties with the X-ray source, is bright and has a complex morphology, with at least three components (with flux ranging from $\sim 0.02$ to $\sim 3 \mathrm{Jy}$ ). This, combined with the characteristics of the radio spectrum (see Figure 2, right), suggests an AGN nature for this source. 


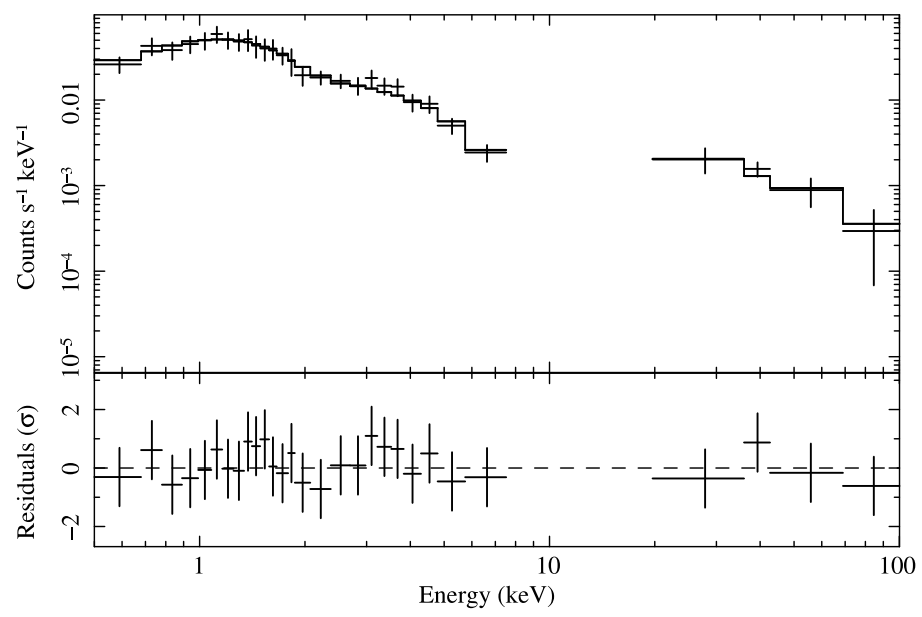

Figure 1: XRT/IBIS broad-band spectrum of IGR J18249-3243 fitted with a simple power law (upper panel); residuals to this model are in units of $\sigma$ (lower panel).
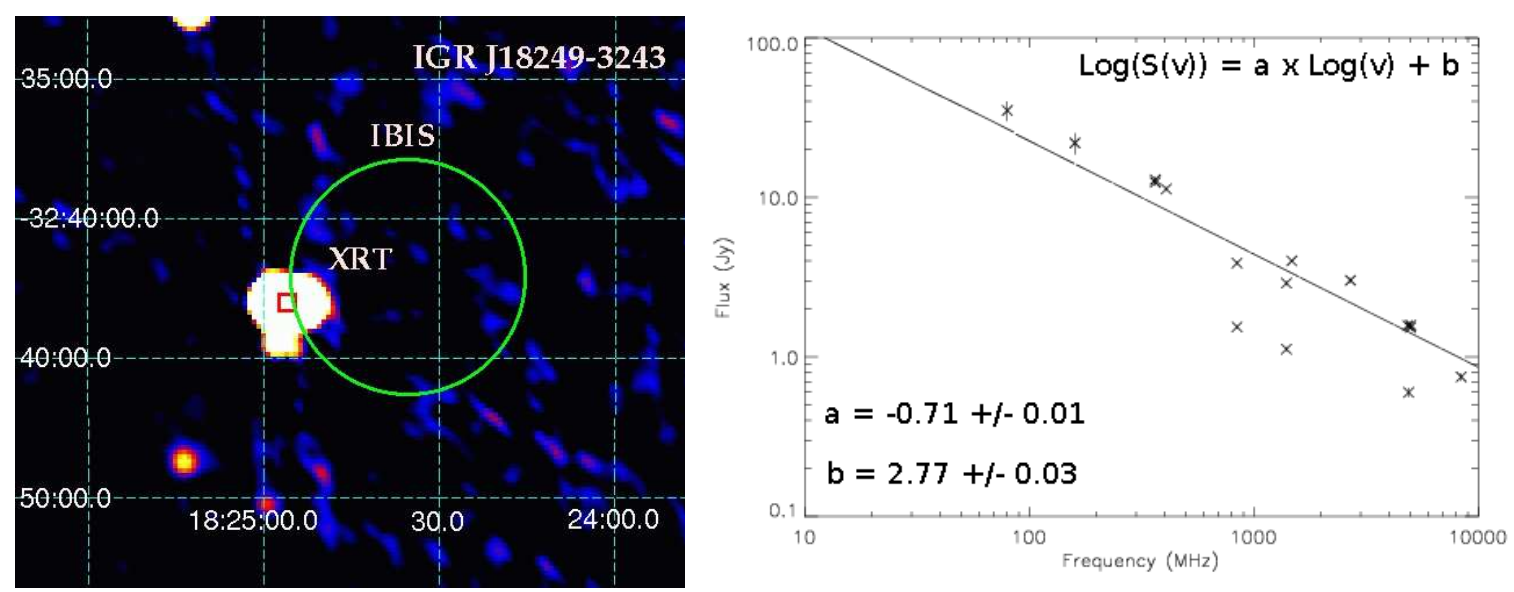

Figure 2: NVSS image (left) and radio spectrum of IGR J18249-3243 fitted with $\log (S(v))=a \times \log (v)+$ $b$ (right). The green circle describes the IBIS uncertainty, while the XRT position is given by the red box.

The X-ray/radio to optical ratios $\geq 0.7$ and $\geq 100$ support the AGN classification (Stocke et al. 1991) and also indicate that the source is a radio loud AGN (Kellerman et al. 1989).

\section{IGR J19443+2117}

For this source we find a 2MASS counterpart, within the XRT position (see Table 2), having magnitudes $J>14.53, H>13.36$ and $K=13.980 \pm 0.07$. Using the 2MASS conversion factors (Skrutskie et al. 2006), the K-band infrared flux is $6.5 \times 10^{-13} \mathrm{erg} \mathrm{cm}^{-2} \mathrm{~s}^{-1}$. This infrared source has no counterpart in the USNO-B1.0 catalogue and Swift/UVOT images, implying a dim flux at optical/UV frequencies. The Galactic colour excess in the source direction is $E(B-V)=2.59$, corresponding to a column density of $1.4 \times 10^{22} \mathrm{~cm}^{-2}$ (Predehl \& Schmitt 1995), significantly higher than the Galactic column density value, but compatible with the total absorption measured in this source (see Table 2). The XRT and IBIS data strongly require, at more than $99.99 \%$ confidence 


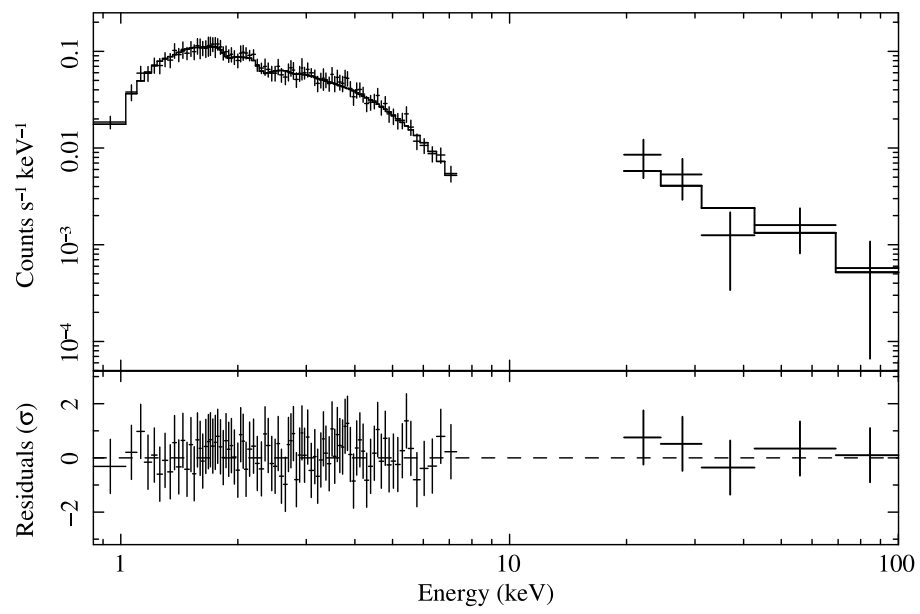

Figure 3: XRT/IBIS broad-band spectrum of IGR J19443+2117 fitted with an absorbed power law (upper panel); residuals to this model are in units of $\sigma$ (lower panel).
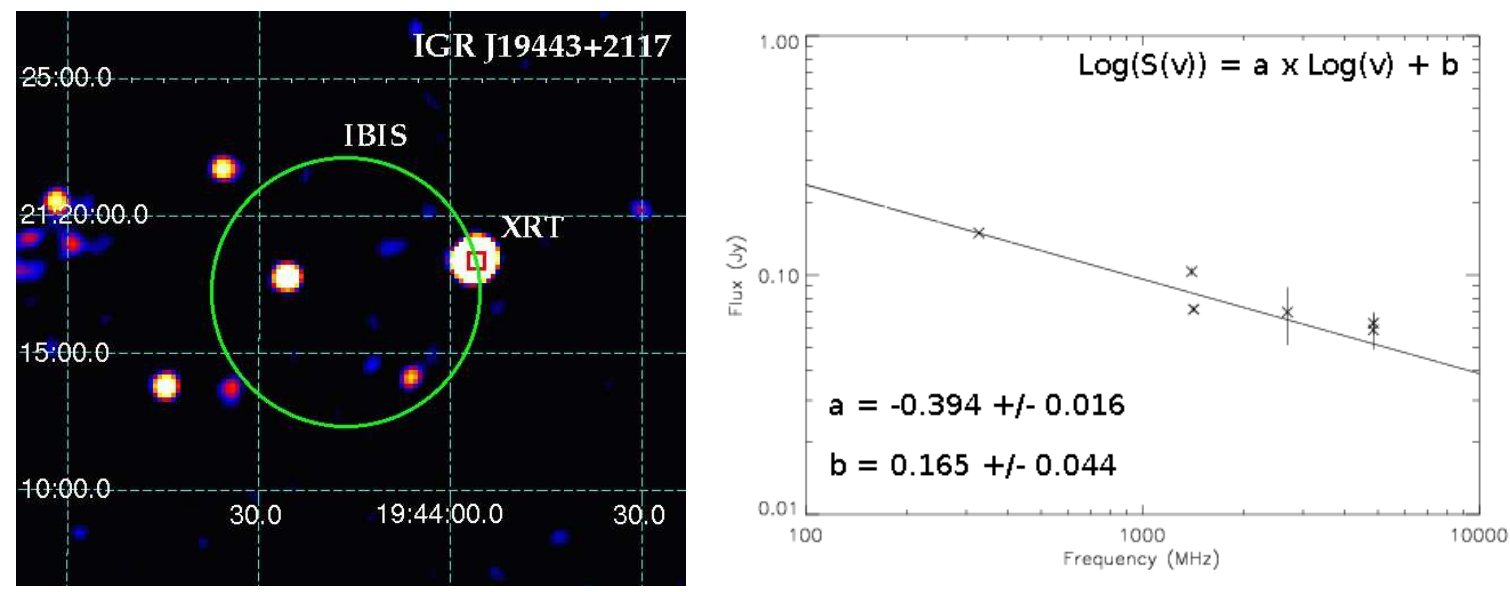

Figure 4: NVSS image (left) and radio spectrum of IGR J19443+2117 fitted with $\log (S(v))=a \times \log (v)+$ $b$ (right). The green circle describes the IBIS uncertainty, while the XRT position is given by the red box.

level according to the F-test, absorption in excess of the Galactic value (see Table 2). The best-fit model, shown in Figure 3, provides a photon index $\Gamma \sim 2$, which is compatible with values typically found in AGNs. The NVSS radio image, plotted in Figure 4 (left), shows the radio counterpart of IGR J19443+2117, which coincides with the XRT position and is characterized by a simple structure. This source is moderately bright (with a flux of $\sim 0.1 \mathrm{Jy}$ at $1.4 \mathrm{GHz}$ ) and shows a flat radio spectrum (see Figure 4, right).

The fact that IGR J19443+2117 is very dim at optical/UV frequencies makes its classification difficult; as the source is the closest of the three to the Galactic plane and with the least information available, its classification remains uncertain. However, based on our multiwavelength study, we propose it as an AGN candidate. 

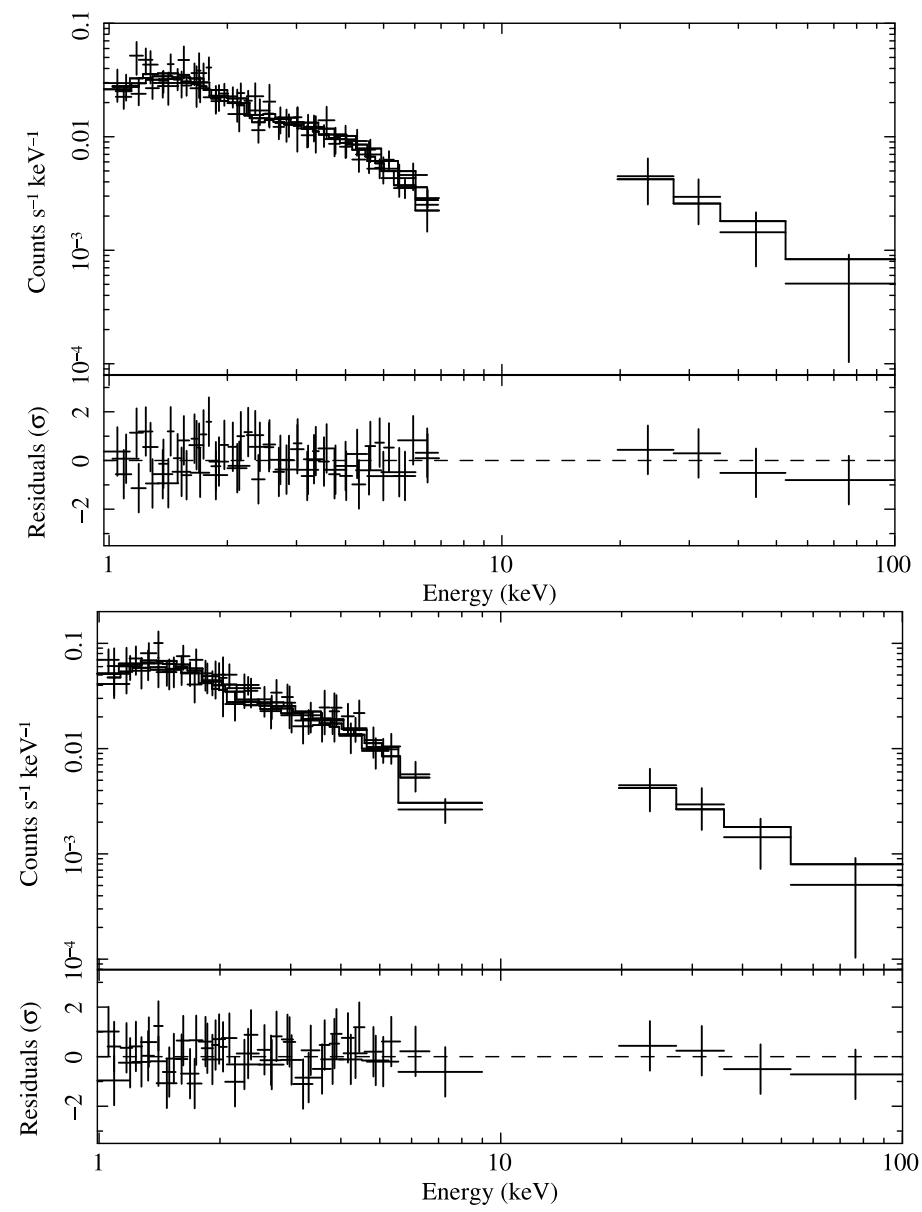

Figure 5: XRT/IBIS broad-band spectra of IGR J22292+6647 in low state (left) and high state (right) fitted with a simple power law (upper panels); residuals to this model are in units of $\sigma$ (lower panels).

\section{IGR J22292+6647}

The field of IGR J22292+6647 was observed from the Astronomical Observatory of Bologna in Loiano (Italy) with the $1.5 \mathrm{~m}$ "G.D. Cassini" telescope. The R-band photometry of the putative optical counterpart of the IBIS source provides a magnitude $R=19.2 \pm 0.1$. This object is also listed in the 2MASS catalogue with magnitudes $J=16.602 \pm 0.143, H=15.707 \pm 0.163$ and $K=14.683 \pm 0.126$. The location of IGR J22292 +6647 in the $\mathrm{J}-\mathrm{H}$ versus $\mathrm{H}-\mathrm{K}$ colour=colour diagram is fully compatible with an AGN nature for this source (Spinoglio et al. 1995). The Galactic colour excess measured along its line of sight is $E(B-V)=1.089$ and corresponds to a hydrogen column density of $0.6 \times 10^{22} \mathrm{~cm}^{-2}$ (Predehl \& Schmitt 1995), compatible with the value reported in Table 2 . The corrected value $\mathrm{R}-\mathrm{K}$ is 2.1 , fully compatible with typical AGN colours. The $R$ magnitude, estimated using the conversion factors of Fukugita et al. (1995), gives a flux of $8.9 \times 10^{-13} \mathrm{erg} \mathrm{cm}^{-2} \mathrm{~s}^{-1}$.

The X-ray data of this source show a flux variability of $\sim 50 \%$ during the XRT observations; therefore, we combined measurements with similar count rates to provide low (LS) and high (HS) states for comparison with the average IBIS spectrum. Despite the changes in flux, no differences 

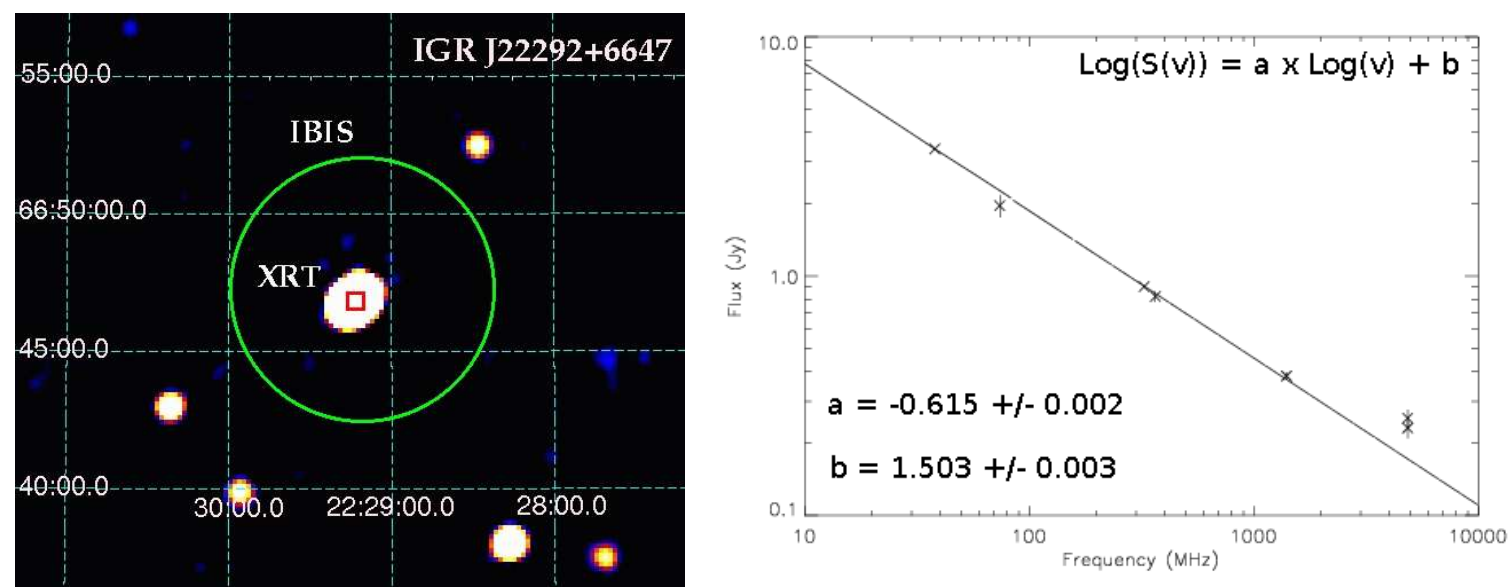

Figure 6: NVSS image (left) and radio spectrum of IGR J22292+6647 fitted with $\log (S(v))=a \times \log (v)+$ $b$ (right). The green circle describes the IBIS uncertainty, while the XRT position is given by the red box.

in shape are observed in the broad-band spectra of the LS and HS, which are well fitted with a simple power law (see Figure 5) having, within uncertainties, the same photon index (see Table 2). The low value of the IBIS/XRT cross-calibration constant confirms the source variability.

Also in this case the radio counterpart coincides with the XRT position, as shown in the NVSS image plotted in Figure 6, left, and has one component with a flux at $1.4 \mathrm{GHz}$ of $\sim 0.4 \mathrm{Jy}$. This radio source has possibly a complex morphology (asymmetric double?) and has a flat spectrum (shown in Figure 6, right); all these characteristics are indicative of an AGN nature for IGR J22292+6647 (Stocke et al. 1991). The X-ray and radio to optical flux ratios of 8 and 476 respectively, confirm this and further suggest it might be a radio loud AGN (Kellerman et al. 1989).

\section{References}

[1] Kalberla, P. M. W., Burton, W. B., Hartmann, D., et al. 2005, A\&A, 440, 775

[2] Monet, D. G., Levine, S. E., Canzian, B.; Ables, et al. 2003, AJ, 125, 984

[3] Skrutskie, M. F., Cutri, R. M., Stiening, R., et al. 2006, AJ, 131, 1163

[4] Schlegel, D. J., Finkbeiner, D. P., \& Davis, M. 1998, ApJ, 500, 525

[5] Predehl, P., \& Schmitt, J. H. M. M. 1995, A\&A, 293, 889

[6] Masetti, N., Parisi, P., Palazzi, E., 2008, arXiv:0811.4085

[7] Fukugita, M., Shimasaku, K., \& Ichikawa, T. 1995, PASP, 107, 945

[8] Condon, J. J., Cotton, W. D., Greisen, E. W., et al. 1998, AJ, 115, 1693

[9] Stocke, J. T., Morris, S. L., Gioia, I. M., et al. 1991, ApJS, 76, 813

[10] Kellerman, K. I., Sramek, R., Schmidt, M., Shaffer, D. B., \& Green, R. et al. 1989, AJ, 98, 1195

[11] Spinoglio, L., Malkan, M. A., Rush, B., Carrasco, L., Recillas-Cruz, E., et al. 1995, ApJ, 453, 616 